\title{
Supraclavicular and/or celiac lymph node metastases from thoracic esophageal squamous cell carcinoma did not compromise survival following neoadjuvant chemoradiotherapy and surgery
}

\author{
Won Kyung Cho ${ }^{1, *}$, Dongryul Oh ${ }^{1, *}$, Yong Chan Ahn ${ }^{1}$, Young Mog Shim², Jae Ill Zo \\ Jong-Mu Sun ${ }^{3}$, Myung-Ju Ahn ${ }^{3}$ and Keunchil Park ${ }^{3}$ \\ ${ }^{1}$ Department of Radiation Oncology, Samsung Medical Center, Sungkyunkwan University School of Medicine, Gangnam-gu, \\ Seoul, Korea \\ 2 Department of Thoracic and Cardiovascular Surgery, Samsung Medical Center, Sungkyunkwan University School of \\ Medicine, Gangnam-gu, Seoul, Korea \\ 3 Medicine (Division of Hematology-Oncology), Samsung Medical Center, Sungkyunkwan University School of Medicine, \\ Gangnam-gu, Seoul, Korea \\ * These authors have equally contributed to this manuscript as the first authors \\ Correspondence to: Yong Chan Ahn, email: ycahn.ahn@samsung.com,ahnyc@skku.edu \\ Keywords: esophageal cancer, neoadjuvant chemoradiotherapy, supraclavicular lymph node, celiac lymph node, staging \\ Received: May 11,2016 Accepted: September 16, $2016 \quad$ Published: September 22, 2016
}

\section{ABSTRACT}

This study is to evaluate the prognostic significance of supraclavicular and/or celiac lymph node (LN) metastases in locally advanced thoracic esophageal squamous cell carcinoma (ESCC) patients treated with neoadjuvant chemoradiotherapy (NACRT) and surgery. Among the total 199 patients, 75 (37.7\%) had supraclavicular and/or celiac LN metastasis. Surgery was performed following NACRT in 168 patients $(84.4 \%)$. After the median 18.7 (1.0-147.2) months' follow-up, 2-year rates of progressionfree survival (PFS) and overall survival (OS) in all patients were $48.1 \%$ and $65.7 \%$, respectively. In multivariate analyses, negative surgical margin $(p<0.001)$, урт0 stage $(p=0.004)$, and ypNO stage $(p=0.020)$ were significantly favorable factors for PFS, and negative surgical margin $(p<0.001)$ was the only significantly favorable factor for OS. Metastasis to the supraclavicular and/or celiac LNs was significant factor neither for PFS $(p=0.311)$ nor OS $(p=0.515)$. Supraclavicular and/or celiac LN metastasis did not compromise the clinical outcomes following NACRT and surgery.

\section{INTRODUCTION}

The prognosis of resectable esophageal cancer remains still unsatisfactory with 5 -year overall survival (OS) rates of $15 \% \sim 34 \%[1,2]$. A multicenter randomized trial demonstrated that the addition of neoadjuvant chemoradiotherapy (NACRT) and surgery improved OS at 5 years when compared to surgical resection alone in patients with resectable stage (T2-3N0-1M0) (47\% vs. $34 \%, p=0.003$ ) [3]. Several meta-analyses also concluded that NACRT and surgery showed survival benefit when compared to surgery with or without neoadjuvant chemotherapy in locally advanced esophageal cancer $[4$, 5]. Thus, NACRT and surgery has been now recommended as the standard treatment in resectable locally advanced esophageal cancer.

Esophageal cancer can spread bidirectionally through the lymphatics to reach remote locations ranging from the cervical to abdominal lymph nodes (LNs). The $6^{\text {th }}$ edition of American Joint Committee on Cancer (AJCC) staging system [6] defined the regional and non-regional LNs based on the primary tumor location: for thoracic esophageal cancer, upper periesophageal, subcarinal, and lower periesophageal LNs were regional LNs; while cervical and celiac LNs were non-regional LNs. Cervical $\mathrm{LN}$ metastasis from upper thoracic esophageal cancer and celiac LN metastasis from lower thoracic esophageal cancer were classified as M1a stage considering the distance between the primary tumor and metastatic LNs, however, metastases to more distantly located LNs were 
Table 1: Patients' characteristics $(N=199)$

\begin{tabular}{|l|l|}
\hline Characteristics & Number of patients \\
\hline Gender & \\
\hline Male & $190(95.5 \%)$ \\
\hline Female & $9(4.5 \%)$ \\
\hline Median age (range) & $62(37 \sim 80)$ years \\
\hline ECOG performance status & \\
\hline 0 & $12(6 \%)$ \\
\hline 1 & $182(91.5 \%)$ \\
\hline 2 & $4(2.0 \%)$ \\
\hline 3 & $1(0.5 \%)$ \\
\hline Endoscopic ultrasonography done & $116(58.3 \%)$ \\
\hline Tumor location & \\
\hline Upper thoracic & $92(46.2 \%)$ \\
\hline Middle thoracic & $62(31.2 \%)$ \\
\hline Lower thoracic & $45(22.6 \%)$ \\
\hline cT stage & \\
\hline cT1 & $6(3.0 \%)$ \\
\hline cT2 & $39(19.6 \%)$ \\
\hline cT3 & $149(74.9 \%)$ \\
\hline cT4 & $5(2.5 \%)$ \\
\hline cN stage & $11(5.5 \%)$ \\
\hline cN0 & $188(94.5 \%)$ \\
\hline cN+ & \\
\hline cM stage according to the $6^{\text {th }}$ AJCC system & $124(62.3 \%)$ \\
\hline cM0 & $54(27.1 \%)$ \\
\hline cM1a & $21(10.5 \%)$ \\
\hline cM1b & \\
\hline A $10 \%$ \\
\hline
\end{tabular}

Abbreviations: ECOG, Eastern Cooperative Oncology Group; AJCC, American Joint Committee on Cancer

classified as M1b together with distant organ metastasis. The $7^{\text {th }}$ edition of AJCC staging system re-defined the regional LNs as any periesophageal LN from cervical to celiac LN and removed M1a and M1b classifications, but incorporated the numbers of metastatic LN instead [7].

The previous clinical trials, which established NACRT and surgery as the current standard for locally advanced esophageal cancer, mostly included the patients with $\mathrm{cM} 0$, but not $\mathrm{cM} 1 \mathrm{a} / \mathrm{b}$, stage according to the $6^{\text {th }}$ edition of AJCC staging system. The current study aimed to evaluate the prognostic influence of $\mathrm{cM} 1 \mathrm{a} / \mathrm{b}$ by the $6^{\text {th }}$ AJCC system in patients with locally advanced thoracic esophageal squamous cell carcinoma following NACRT and surgery.

\section{RESULTS}

\section{Patient characteristics}

The patients' characteristics are summarized in Table 1 . The median age of all patients was $62(37 \sim 80)$ years and the majority of patients were male (190/199, $95.5 \%$ ). The most frequent location of the primary tumor was the upper thoracic esophagus in 92 patients $(46.2 \%)$, followed by the middle thoracic in $62(31.2 \%)$ and lower thoracic in $45(22.6 \%)$, respectively. Most patients had good to excellent Eastern Cooperative Oncology Group (ECOG) performance status of 0-1 (194/199, 97.5\%). Clinical T stages were cT1 in six patients $(3.0 \%)$, cT2 in $39(19.6 \%)$, cT3 in $149(74.9 \%)$, and cT4 in five $(2.5 \%)$, respectively, and majority had LN metastasis (188/199, $94.5 \%$ ). Had the $6^{\text {th }}$ edition AJCC staging system been applied, $54(27.1 \%)$ and $21(10.6 \%)$ patients would have been classified as cM1a and cM1b stages, respectively. Among the M1a patients, 42 patients had upper thoracic esophageal cancer with supraclavicular LN metastasis and 12 had lower thoracic esophageal cancer with celiac LN metastasis.

\section{Treatment compliance}

Compliance to NACRT was very high: 196 patients $(98.5 \%)$ could complete the planned radiotherapy (RT) 
Table 2: Lymph node dissection and pathologic assessment following surgery $(N=168)$

\begin{tabular}{|l|l|}
\hline Characteristics & Number of patients \\
\hline Surgical procedure & \\
\hline 2 field lymph node dissection & $79(47.0 \%)$ \\
\hline 3 field lymph node dissection & $89(53.0 \%)$ \\
\hline Median number of lymph node dissected & $38(3 \sim 86)$ \\
\hline Surgical margin & \\
\hline Negative & $153(91.1 \%)$ \\
\hline Microscopically positive & $8(4.8 \%)$ \\
\hline Gross residual & $7(4.2 \%)$ \\
\hline ypT stage & \\
\hline ypT0 & $77(45.8 \%)$ \\
\hline ypT1 & $23(13.7 \%)$ \\
\hline ypT2 & $24(14.3 \%)$ \\
\hline ypT3 & $42(25.0 \%)$ \\
\hline ypT4 & $2(1.2 \%)$ \\
\hline ypN stage & \\
\hline ypN0 & $75(44.6 \%)$ \\
\hline ypN1 & $56(33.3 \%)$ \\
\hline ypN2 & $23(13.7 \%)$ \\
\hline ypN3 & $14(8.3 \%)$ \\
\hline Pathologic complete response & \\
\hline Yes & $44(26.2 \%)$ \\
\hline No & $124(73.8 \%)$ \\
\hline &
\end{tabular}

dose $($ median RT dose $=44(3.6 \sim 45)$ Gy); and $191(96.0 \%)$ could complete the 2 cycles of chemotherapy. Surgery was performed in 168 patients $(84.4 \%)$ and the reasons for not undergoing surgery were patients' refusal of surgery in $14(7.0 \%)$, inadequate recovery from NACRT toxicity in six $(3.0 \%)$, identification of disease progression before surgery in five $(2.5 \%)$, development of esophagorespiratory fistula during NACRT in three $(1.5 \%)$, and death while waiting for surgery in three (1.5\%). Among 14 patients who refused surgery, seven received further chemoradiotherapy, while the remaining seven declined any further treatment. Five patients who developed disease progression following NACRT were recommended to receive palliative systemic chemotherapy. Three patients died while waiting for surgery and were assumed to have experienced esophageal perforation and subsequent massive bleeding. The median interval between NACRT completion and surgery was 5.0 (1.6-13.6) weeks. Twoand three-field LN dissections were performed in 79 and 89 patients $(47.0 \%$ and $53.0 \%)$, respectively. Post-surgical mortality occurred in eleven patients (6.5\%): pulmonary complication in nine; anastomosis leakage in one; and tracheoesophageal fistula in one, respectively.

\section{Pathologic assessment}

R0 resection was achieved in 153 patients (91.1\%) (Table 2). Pathologic complete response of primary tumor
(урТ0) was achieved in 77 patients $(45.8 \%)$. The median number of LNs retrieved was 38 (3-86) and 75 patients $(44.6 \%)$ had ypN0 stage. Overall, 44 patients $(26.2 \%)$ achieved pathologic complete response both in the primary tumor and LNs (pCR, ypT0N0).

\section{Clinical outcomes}

After the median 18.7 (1.0-147.2) months' followup on 199 patients, 88 (44.2\%) experienced disease progression and $77(38.7 \%)$ died. The patterns of failure were LR failure in 33 patients (37.5\%), distant metastasis in 32 (36.4\%), and combined LR and distant failure in 23 (26.1\%), respectively. The sites of LR failure in relation to the RT volume were analyzed. Among 56 patients who developed LR failure, 27 patients experienced recurrence inside the radiation volume, 26 did outside the radiation volume, and three did both inside and outside the radiation volume, respectively. The 2-year rates of progression-free survival (PFS) and OS of all patients were $48.1 \%$ and $65.7 \%$, respectively. The clinical outcomes at 2 years of the patients who underwent surgery were significantly better than those who did not: PFS rates were $49.4 \%$ and $37.3 \%(p=0.004)$; and OS rates were $68.2 \%$ and $45.8 \%$ $(p=0.001)$, respectively (Figure 1). Among 168 patients who underwent surgery, 2-year rates of PFS and OS based on yp-stages were $58.8 \%$ and $79.8 \%$ in ypT0N0, $61.0 \%$ and $76.3 \%$ in ypT0N+, $48.7 \%$ and $66.3 \%$ in ypT $+\mathrm{N} 0$, and 

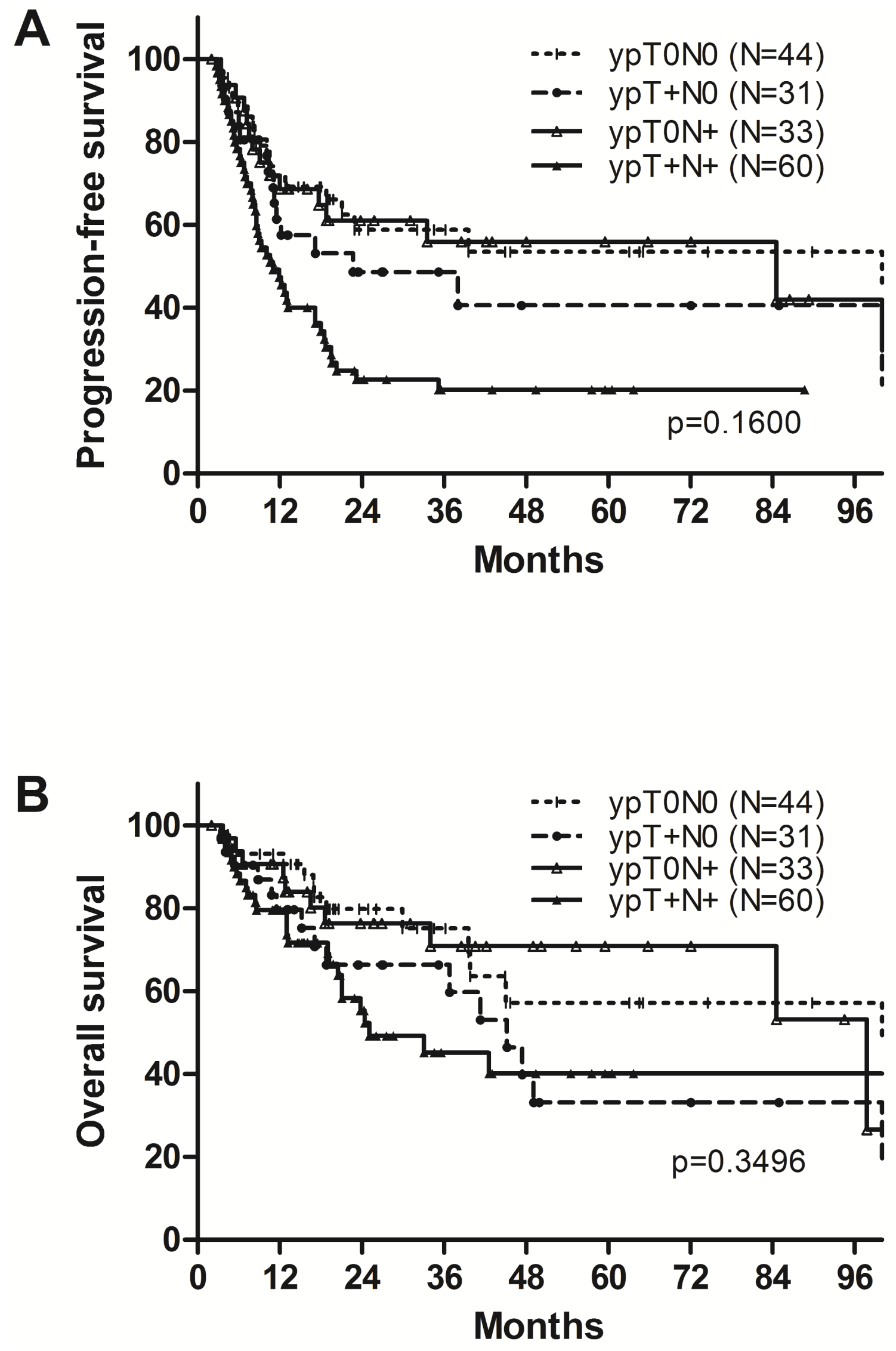

Figure 1: A. Progression-free survival and B. overall survival of all patients. 
Table 3: Prognostic factors by univariate analyses $(N=168)$

\begin{tabular}{|c|c|c|c|c|c|c|}
\hline Factors & 2yr-LRC & $p$ & 2yr-PFS & $p$ & $2 \mathrm{yr}-\mathrm{OS}$ & $p$ \\
\hline Gender & & 0.643 & & 0.778 & & 0.280 \\
\hline Male $(N=159)$ & $60.8 \%$ & & $43.8 \%$ & & $69.0 \%$ & \\
\hline Female $(N=9)$ & $57.1 \%$ & & $44.4 \%$ & & $55.6 \%$ & \\
\hline Age & & 0.122 & & 0.699 & & 0.017 \\
\hline$\leq 60$ years $(N=76)$ & $51.7 \%$ & & $41.0 \%$ & & $76.4 \%$ & \\
\hline$>60$ years $(N=92)$ & $69.7 \%$ & & $46.4 \%$ & & $61.1 \%$ & \\
\hline Tumor location & & 0.454 & & 0.131 & & 0.121 \\
\hline Upper thoracic $(N=76)$ & $64.0 \%$ & & $46.3 \%$ & & $69.2 \%$ & \\
\hline Middle thoracic $(N=52)$ & $58.6 \%$ & & $34.0 \%$ & & $61.2 \%$ & \\
\hline Lower thoracic $(N=40)$ & $55.8 \%$ & & $51.2 \%$ & & $76.2 \%$ & \\
\hline cT stage & & 0.040 & & 0.792 & & 0.532 \\
\hline cT1-2 $(N=38)$ & $47.0 \%$ & & $43.4 \%$ & & $66.0 \%$ & \\
\hline cT3-4 $(N=130)$ & $65.8 \%$ & & $43.9 \%$ & & $68.8 \%$ & \\
\hline cN stage & & 0.868 & & 0.630 & & 0.592 \\
\hline $\mathrm{cN} 0(N=9)$ & $63.5 \%$ & & $55.6 \%$ & & $55.6 \%$ & \\
\hline $\mathrm{cN}+(N=159)$ & $60.4 \%$ & & $43.0 \%$ & & $69.0 \%$ & \\
\hline cM stage ( $6^{\text {th }}$ AJCC system $)$ & & 0.481 & & 0.311 & & 0.515 \\
\hline $\mathrm{cM} 0(N=104)$ & $64.0 \%$ & & $49.5 \%$ & & $69.3 \%$ & \\
\hline $\mathrm{cM} 1 \mathrm{a} / \mathrm{b}(N=64)$ & $56.0 \%$ & & $36.3 \%$ & & $67.4 \%$ & \\
\hline Lymph node dissection & & 0.074 & & 0.486 & & 0.890 \\
\hline 2 fields $(N=79)$ & $53.1 \%$ & & $40.9 \%$ & & $67.8 \%$ & \\
\hline 3 fields $(N=89)$ & $67.0 \%$ & & $46.1 \%$ & & $68.8 \%$ & \\
\hline ypT stage & & 0.001 & & 0.001 & & 0.011 \\
\hline урТ0 $(N=77)$ & $77.5 \%$ & & $60.1 \%$ & & $78.3 \%$ & \\
\hline ypT1-4 $(N=91)$ & $45.4 \%$ & & $30.4 \%$ & & $59.6 \%$ & \\
\hline ypN stage & & 0.021 & & 0.021 & & 0.472 \\
\hline ypN0 $(N=75)$ & $71.4 \%$ & & $54.1 \%$ & & $74.4 \%$ & \\
\hline $\mathrm{ypN}+(N=93)$ & $51.7 \%$ & & $35.8 \%$ & & $63.4 \%$ & \\
\hline p-complete response (pCR) & & 0.007 & & 0.014 & & 0.118 \\
\hline $\mathrm{pCR}(N=44)$ & $80.8 \%$ & & $58.8 \%$ & & $79.8 \%$ & \\
\hline Non-pCR $(N=124)$ & $53.3 \%$ & & $38.4 \%$ & & $64.3 \%$ & \\
\hline Adjuvant chemotherapy & & 0.374 & & 0.310 & & 0.104 \\
\hline Yes $(N=36)$ & $64.3 \%$ & & $48.2 \%$ & & $73.4 \%$ & \\
\hline $\operatorname{No}(N=132)$ & $52.5 \%$ & & $42.7 \%$ & & $66.7 \%$ & \\
\hline Surgical margin & & $<0.001$ & & $<0.001$ & & $<0.001$ \\
\hline Negative $(N=153)$ & $63.1 \%$ & & $48.1 \%$ & & $72.7 \%$ & \\
\hline Positive $(N=15)$ & $21.5 \%$ & & 0 & & $19.0 \%$ & \\
\hline
\end{tabular}

Abbreviations: LRC, locoregional control; PFS, progression free survival; OS, overall survival

$22.7 \%$ and $55.4 \%$ in ypT $+\mathrm{N}+$, respectively (Figure 2 ).

The probable prognosticators were analyzed by the univariate analyses in 168 patients who underwent surgery. The factors included gender, age, primary tumor location, cT stage, $\mathrm{cN}$ stage, $6^{\text {th }}$ AJCC M stage, surgical procedure, ypT stage, ypN stage, pCR, adjuvant chemotherapy, and surgical margin (Table 3). The significantly favorable factors on loco-regional control (LRC) at 2 years were negative surgical margin $(p<0.001), \operatorname{ypT} 0(p=0.001)$, cT1-2 $(p=0.040), \operatorname{pCR}(p=0.007)$, and ypN0 $(p=0.021)$. Favorable factors on PFS at 2 years were negative surgical margin $(p<0.001)$, ypT0 $(p=0.001), \operatorname{pCR}(p=0.014)$, and ypN0 $(p=0.021)$, and those on OS were negative surgical margin $(p<0.001), \operatorname{ypT0}(p=0.011)$, and age of 60 years or younger $(p=0.017)$. In the multivariate analysis, negative surgical margin (HR 0.263; 95\% CI 

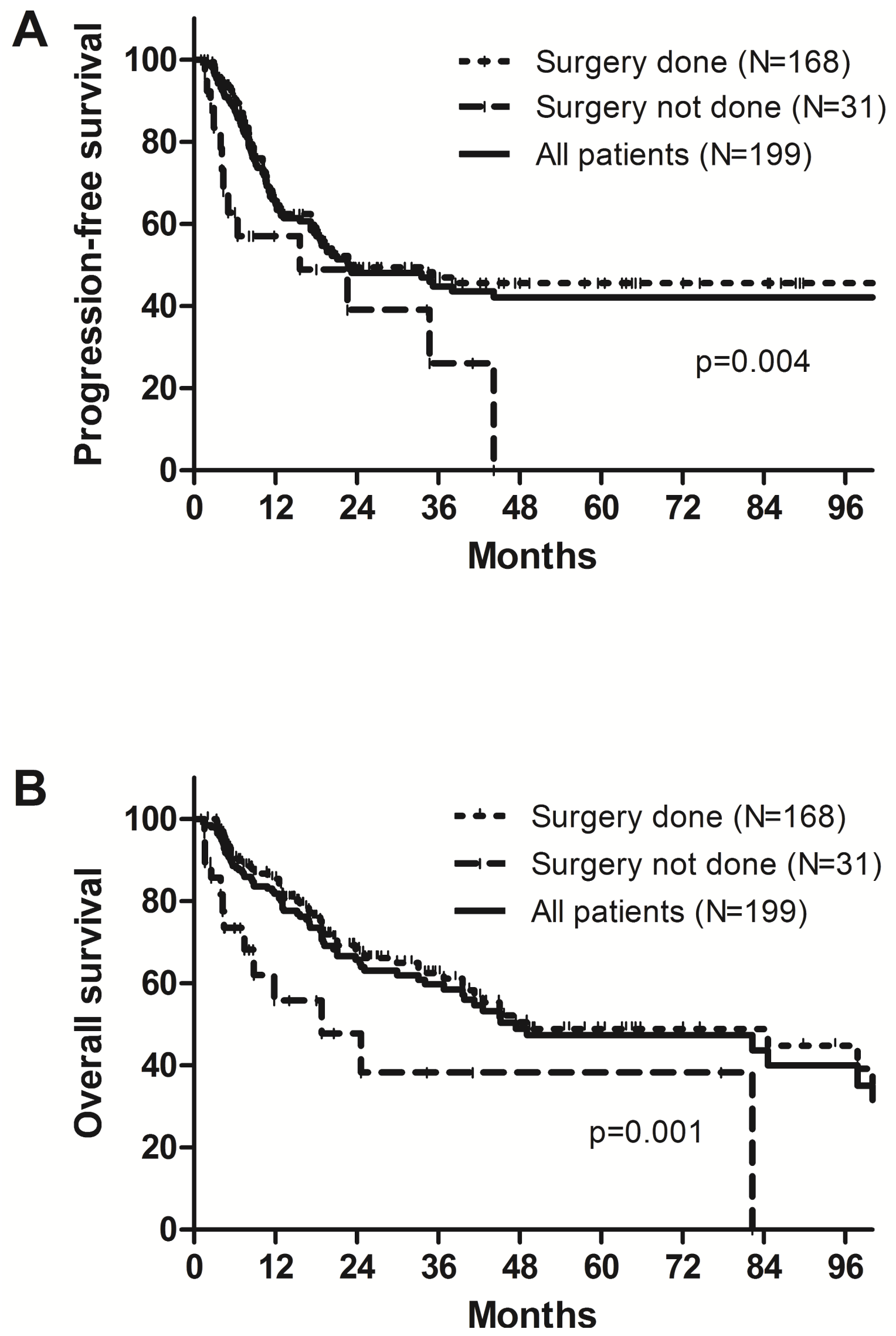

Figure 2: A. Progression-free survival and B. overall survival according to ypT and ypN stages. 
Table 4: Prognostic factors by multivariate analyses $(N=168)$

\begin{tabular}{|c|c|c|c|c|c|c|}
\hline \multirow{2}{*}{ Factors } & \multicolumn{2}{|c|}{ Locoregional control } & \multicolumn{2}{|c|}{ Progression-free survival } & \multicolumn{2}{|c|}{ Overall survival } \\
\hline & HR $(95 \%$ CI) & $p$ & HR $(95 \%$ CI $)$ & $p$ & HR $(95 \%$ CI) & $p$ \\
\hline Gender & 0.863 & \multirow{2}{*}{0.832} & 0.852 & \multirow{2}{*}{0.751} & 0.493 & \multirow{2}{*}{0.215} \\
\hline Male vs. female & $(0.221-3.370)$ & & $(0.316-2.294)$ & & $(0.161-1.508)$ & \\
\hline Age & 1.496 & \multirow{2}{*}{0.198} & 1.086 & \multirow{2}{*}{0.708} & 0.582 & \multirow{2}{*}{0.582} \\
\hline$\leq 60$ years vs. $>60$ years & $(0.810-2.762)$ & & $(0.706-1.671)$ & & $(0.333-1.019)$ & \\
\hline \multicolumn{7}{|l|}{ Location } \\
\hline \multirow[t]{2}{*}{ Upper vs. lower } & 1.016 & \multirow[b]{2}{*}{0.973} & 1.132 & \multirow[b]{2}{*}{0.734} & 1.24 & \multirow[b]{2}{*}{0.65} \\
\hline & $(0.403-2.559)$ & & $(0.554-2.314)$ & & $(0.489-3.144)$ & \\
\hline \multirow[t]{2}{*}{ Middle vs. lower } & 1.316 & \multirow{2}{*}{0.506} & 2.031 & \multirow{2}{*}{0.025} & 2.31 & \multirow{2}{*}{0.047} \\
\hline & $(0.586-2.959)$ & & $(1.093-3.774)$ & & $(1.011-5.277)$ & \\
\hline cT stage & 2.305 & \multirow{2}{*}{0.013} & 1.125 & \multirow{2}{*}{0.647} & 1.183 & \multirow{2}{*}{0.618} \\
\hline cT1-2 vs. cT3-4 & $(1.194-4.448)$ & & $(0.679-1.865)$ & & $(0.611-2.290)$ & \\
\hline cN stage & 1.661 & \multirow{2}{*}{0.461} & 0.937 & \multirow{2}{*}{0.907} & 1.49 & \multirow{2}{*}{0.494} \\
\hline cN0 vs. cN+ & $(0.431-6.402)$ & & $(0.317-2.773)$ & & $(0.475-4.675)$ & \\
\hline $\begin{array}{l}\text { cM stage (6th AJCC } \\
\text { system) }\end{array}$ & 0.788 & \multirow{2}{*}{0.484} & 0.764 & \multirow{2}{*}{0.267} & 0.742 & \multirow{2}{*}{0.307} \\
\hline cM0 vs. cM1a/b & $(0.404-1.537)$ & & $(0.475-1.229)$ & & $(0.418-1.317)$ & \\
\hline Lymph node dissection & 2.438 & \multirow[b]{2}{*}{0.044} & 1.333 & \multirow[b]{2}{*}{0.326} & 1.16 & \multirow[b]{2}{*}{0.667} \\
\hline 2 fields vs 3 fields & $(1.024-5.807)$ & & $(0.751-2.366)$ & & $(0.590-2.282)$ & \\
\hline ypT stage & 0.368 & & 0.413 & & 0.518 & \\
\hline ypT0 vs. ypT1-4 & $(0.164-0.828)$ & 0.016 & $(0.228-0.748)$ & 0.004 & $(0.240-1.118)$ & 0.094 \\
\hline ypN stage & 0.558 & 0167 & 0.49 & בח & 0.694 & A 322 \\
\hline ypN0vs. ypN1-3 & $(0.244-1.276)$ & 0.101 & $(0.269-0.892)$ & 0.02 & $(0.336-1.431)$ & 10.322 \\
\hline $\begin{array}{ll}\text { p-complete response } \\
(\mathrm{pCR})\end{array}$ & 0.818 & 0.772 & 0.574 & 0.228 & 0.745 & 0.6 \\
\hline pCR vs. Non-pCR & $(0.209-3.193)$ & & $(0.233-1.416)$ & & $(0.249-2.235)$ & \\
\hline Adjuvant chemotherapy & 0.824 & & 1.479 & & 1.51 & \\
\hline Yes vs. no & $(0.394-1.722)$ & 0.606 & $(0.842-2.600)$ & 0.174 & $(0.710-3.212)$ & 0.284 \\
\hline Surgical margin & 0.176 & $<0.001$ & 0.263 & $<0.001$ & 0.187 & $<0.001$ \\
\hline Negative vs. positive & $(0.061-0.510)$ & & $(0.132-0.522)$ & & $(0.086-0.404)$ & \\
\hline
\end{tabular}

Abbreviations; HR, hazard ratio; $\mathrm{CI}$, confidential interval

0.132-0.522; $p<0.001$ ), ypT0 stage (HR, 0.413; 95\% CI $0.228-0.748 ; p=0.004$ ), and ypN0 stage (HR 0.490; 95\% CI $0.269-0.892 ; p=0.020)$ were statistically significant favorable prognostic factors for PFS at 2 years. Negative surgical margin (HR 0.187; 95\% CI 0.086-0.404; $p<$ $0.001)$ was the only favorable factor for OS at 2 years (Table 4).

\section{Significance of supraclavicular and/or celiac LN metastasis}

Among all, the patients having metastasis to the supraclavicular and/or celiac LNs (cMla/b group) showed numerically lower, but not significant, PFS (40.1\% vs. $53.5 \%, p=0.204)$ and $\mathrm{OS}(62.5 \% v s .68 .4 \%, p=0.362)$ at 2 years, when compared to those having regional LN metastasis (cM0 group). Among 168 patients who underwent surgery, pCR rate was numerically higher in 

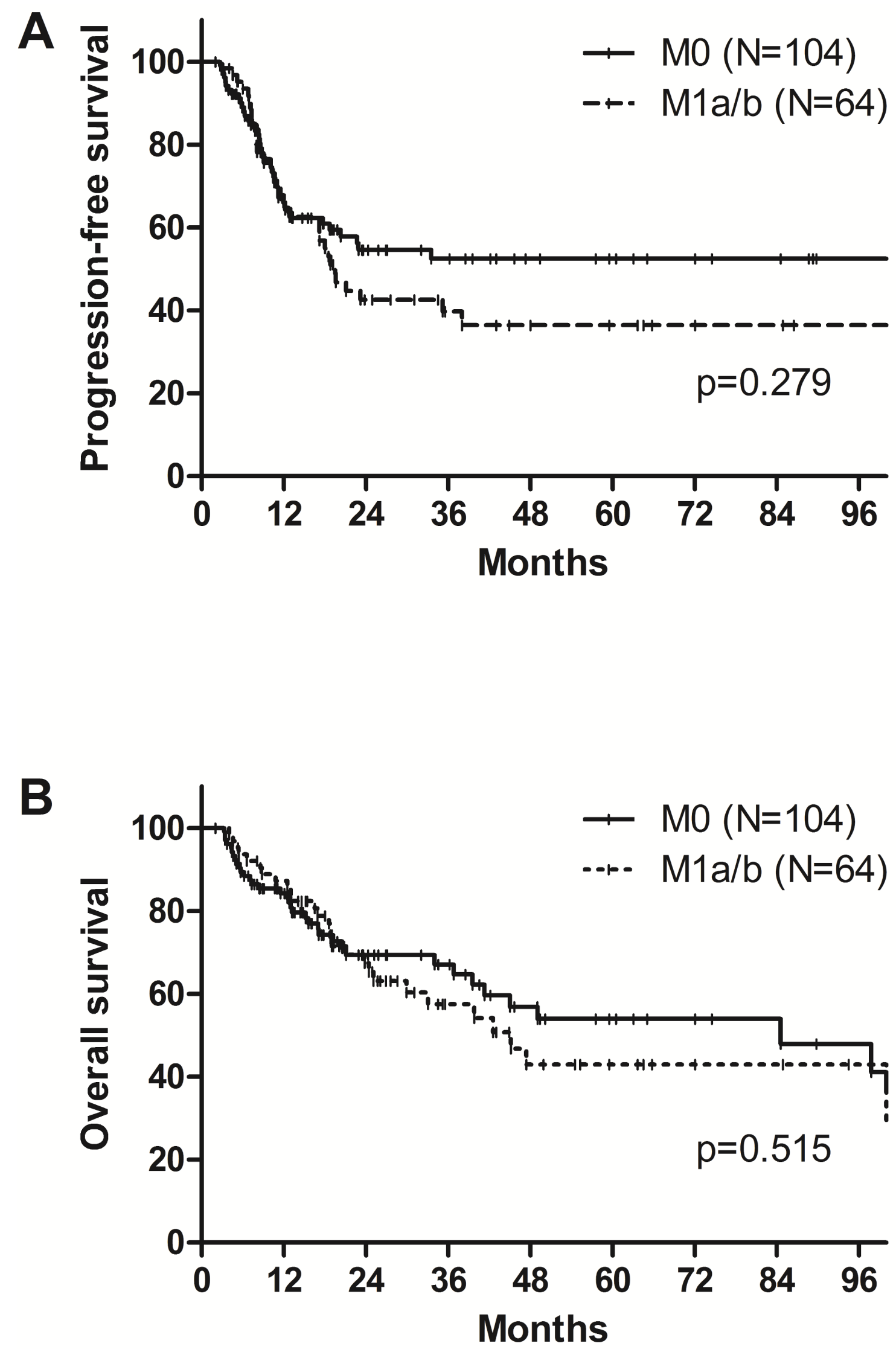

Figure 3: A. Progression-free survival and B. overall survival according to supraclavicular and/or celiac lymph node metastasis $(\mathrm{cM} 1 \mathrm{a} / \mathrm{b})$ among patients who underwent surgery. 
cM0 group, but not significant $(29.8 \%$ vs. $20.3 \%, p=$ $0.174)$, and the same trends were shown on PFS $(42.5 \%$ vs. 54.6\%, $p=0.279)$ and OS (67.4\% vs. $69.3 \%, p=$ 0.515 ) at 2 years (Figure 3 ).

\section{DISCUSSION}

Supraclavicular and celiac LNs were defined as non-regional LNs of thoracic esophageal cancer in the $6^{\text {th }}$ AJCC staging system. However, a number of evidence had suggested that supraclavicular and/or celiac LN metastasis does not compromise prognosis compared to other regional LN metastasis [8-11]. Thus, in the $7^{\text {th }}$ AJCC staging system, discrimination of cervical and celiac LNs as non-regional LNs was abandoned. However, the prognostic impact of supraclavicular and/or celiac LN metastasis in the setting of NACRT and surgery has not been properly addressed yet, since most previous trials, including the CROSS trial, did not include the patients with $\mathrm{cM} 1 \mathrm{a} / \mathrm{b}$ stage by the $6^{\text {th }}$ AJCC staging system $[3,12$, 13]. The current study revealed that the supraclavicular and/or celiac LN metastasis did not compromise survival outcomes in the patients who tolerated tri-modality treatment of NACRT and surgery.

Several prognostic factors have been suggested in esophageal cancer following NACRT and surgery such as tumor location, cT stage, surgical margin status, and pCR rate [14-17]. The present study also confirmed that negative resection margin was a significantly favorable prognostic factor for both PFS and OS in multivariate analysis $(p<0.001)$. pCR rate following NACRT is known as $25 \% \sim 30 \%[3,15,16]$ though its prognostic significance is still controversial $[18,19]$. In the current study, pCR was achieved in $26.2 \%$ and it was not a significant factor on OS at 2 year $(79.8 \%$ vs. $64.3 \%, p=0.118)$. This might be partly explained by the fact that OS at 2 years of patients with pT0N+ and pT+N0 (76.3\% and 66.3\%) were not different from that of patients achieving pCR (79.8\%) (Figure 2).

Recently, the prognostic implication of FDG-PET parameters following NACRT was raised. Our institution previously reported the impact of FDG-PET parameter following NACRT in esophageal cancer patients [20]. In the present study, we could not incorporate the FDG-PET parameters into the prognostic factor analysis because the raw data of FDG-PET parameters were not available in $20 \%$ of the patients, whose FDG-PET CT was done at other hospitals. However, based on the available data in the previous study, SUVmax value reduction rates of $72.1 \%$ in main tumor and $50.7 \%$ in LNs estimated pCR with the best accuracy while total lesion glycolysis did not.

The clinical outcomes by the current study were favorably comparable with those by previous randomized studies, even though $37.7 \%$ of patients in the current study had $\mathrm{M} 1 \mathrm{a} / \mathrm{b}$ disease. The previous randomized studies reported OS rates of $60 \% \sim 67 \%$ at 2 years and $39 \% \sim 47 \%$ at 5 years following NACRT and surgery $[3,12,13]$. In this study, the 2- and 5-year OS rates were $69.3 \%$ and $53.9 \%$, respectively. Furthermore, the rates of $\mathrm{R} 0$ resection and postoperative mortality in hospital or within 30 days were $91.1 \%$ and $6.5 \%$, respectively, which were also comparable with the previous studies $(92.0 \%$ and $5.9 \%$ in the CROSS trial and $93.8 \%$ and $11.1 \%$ in the FFCD 9901 trial, respectively) $[3,13]$.

This study has a few limitations. First, this study included rather a limited number of patients to reach concrete conclusions. However, considering that we included only the patients with squamous cell carcinoma of the thoracic esophagus, who were treated in a highly homogenous manner, 199 patients is not a small number. Squamous cell carcinoma is the most prevalent histologic type in Asian countries such as China, Japan, and Korea. In fact, adenocarcinoma composed of only $2.5 \%$ of the patients who received NACRT for esophageal cancer in our institution. Second, a selection bias might have existed as NACRT and surgery was mainly recommended to those who had favorable performance status and were expected to tolerate large RT volume. In fact, chemotherapy alone before surgery was mainly recommended to the patients who had marginal performance status. Thus, our results might not be applicable to all patients having supraclavicular and/or celiac LN metastasis.

In summary, celiac and/or supraclavicular LN metastasis did not compromise the survival in patients with locally advanced esophageal squamous cell carcinoma who received NACRT and surgery. Further larger prospective studies should be warranted to establish the optimal treatment of thoracic esophageal cancer with celiac and/or cervical LN.

\section{MATERIALS AND METHODS}

\section{Patients}

NACRT and surgery was recommended as the definitive therapy to 201 patients for having locally advanced thoracic esophageal squamous cell carcinoma at the authors' institute from January 2003 till July 2014. Following approval by our institutional review board, we retrospectively reviewed the medical records. After excluding two patients, who abandoned their treatment during NACRT course and were lost to our follow-up, analyses were done on 199. In all patients, endoscopy with biopsy, chest computed tomography (CT), and whole body $18 \mathrm{~F}$-fluorodeoxyglucose positron emission tomography-computed tomography $\left({ }^{18} \mathrm{~F}-\mathrm{FDG}\right.$ PETCT) were performed for diagnosis and staging purpose. Endoscopic ultrasonography (EUS) was performed in 116 patients $(58.3 \%)$. Primary tumor location was allocated 
according to the uppermost involvement of the primary tumor according to the AJCC $7^{\text {th }}$ edition.

\section{Treatment scheme}

RT was to deliver 45 Gy in 25 fractions by 1.8 Gy per fraction over 5 weeks until March of 2009 in 54 patients $(27.1 \%)$ and 44 Gy in 22 fractions by 2.0 Gy per fraction thereafter in $145(72.9 \%)$. All patients received 3-dimensional conformal RT (3D-CRT), typically through 3 or 4 coplanar fields using 4-, 6-, or 10-MV photons from linear accelerator. Primary tumor and metastatic LNs were delineated as the gross tumor volume (GTV) based on CT, PET-CT, and endoscopic findings. The clinical target volume (CTV) of primary tumor included primary GTV plus $2 \sim 3 \mathrm{~cm}$ margins in the craniocaudal directions and $0.5 \mathrm{~cm}$ margin in the circumferential direction. The nodal CTV was delineated by putting $1 \mathrm{~cm}$ margin in all directions from the nodal GTV. Elective irradiation to the supraclavicular and/or celiac LNs was not employed in the current study. The planning target volume (PTV) was defined as $0.5 \sim 0.7 \mathrm{~cm}$ margin in all directions from the CTV to account for the respiratory motion and daily setup errors.

Two cycles of intravenous chemotherapy (5-flurouracil (5-FU) 1,000 mg/m²/day for 4 consecutive days plus cisplatin $60 \mathrm{mg} / \mathrm{m}^{2} /$ day on the $1^{\text {st }}$ day) were administered at 3 weeks' interval, and the $1^{\text {st }}$ cycle was to planned on the $1^{\text {st }}$ day of RT. Tumor response and resectability was re-evaluated before surgical resection by chest CT and FDG PET-CT in 3 4 weeks of NACRT completion.

Esophageal resection through thoracotomy was combined with either two-field or three-field lymphadenectomy. Two-field (thoracic and abdominal) LN dissection was performed mainly in the patients with lower thoracic esophageal cancer while three-field (cervical, thoracic, and abdominal) LN dissection was done mainly in those with upper or mid-thoracic esophageal cancer. Following NACRT, adjuvant chemotherapy or thoracic RT were optionally recommended to the patients with good performance status and risk factors based on surgical and pathological findings: 3 cycles of 5-FU and cisplatin chemotherapy was delivered in 36 patients $(21.4 \%)$; 20 Gy in 10 fractions thoracic RT was in $13(7.7 \%)$, respectively. Usual indications for adjuvant therapy included multiple pathologic lymph node metastases, close or positive resection margin, and/or difficulty of dissection experienced at time of surgery due to bulky primary tumor and/or metastatic LN.

\section{Statistical analyses}

The durations of PFS and OS were calculated from the first date of NACRT till the date of any event of recurrence and death or the last follow-up, respectively. The distribution of categorical variables was analyzed by the chi-square test. The survival rates were calculated by the Kaplan-Meier method and were compared by logrank test for univariate analysis. Multivariate analysis was described with $\mathrm{HR}$ and their $95 \% \mathrm{CI}$, derived from the cox proportional hazards model. Two-sided p-values of 0.05 or less were considered statistically significant. SPSS Statistics version 20 (SPSS Inc., an IBM Company, Chicago, IL) was used for the analysis.

\section{CONFLICTS OF INTEREST}

The authors declare no conflicts of interest

\section{REFERENCES}

1. Demeester SR. Epidemiology and biology of esophageal cancer. Gastrointest Cancer Res. 2009; 3:S2-5.

2. Allum WH, Stenning SP, Bancewicz J, Clark PI and Langley RE. Long-term results of a randomized trial of surgery with or without preoperative chemotherapy in esophageal cancer. J Clin Oncol. 2009; 27:5062-5067.

3. van Hagen P, Hulshof MC, van Lanschot JJ, Steyerberg EW, van Berge Henegouwen MI, Wijnhoven BP, Richel DJ, Nieuwenhuijzen GA, Hospers GA, Bonenkamp JJ, Cuesta MA, Blaisse RJ, Busch OR, et al. Preoperative chemoradiotherapy for esophageal or junctional cancer. $\mathrm{N}$ Engl J Med. 2012; 366:2074-2084.

4. Sjoquist KM, Burmeister BH, Smithers BM, Zalcberg JR, Simes RJ, Barbour A, Gebski V and Australasian Gastro-Intestinal Trials G. Survival after neoadjuvant chemotherapy or chemoradiotherapy for resectable oesophageal carcinoma: an updated meta-analysis. Lancet Oncol. 2011; 12:681-692.

5. Fan M, Lin Y, Pan J, Yan W, Dai L, Shen L and Chen K. Survival after neoadjuvant chemotherapy versus neoadjuvant chemoradiotherapy for resectable esophageal carcinoma: A meta-analysis. Thorac Cancer. 2016; 7:173181.

6. Frederick LG, Page DL, Fleming ID, Fritz AG, Balch CM, Haller DG and Morrow M. AJCC Cancer Staging Manual. New York: Springer; 2002.

7. Edge S, Byrd DR, Compton CC, Fritz AG, Greene FL and Trotti A. AJCC Cancer Staging Manual. 7th ed. New York: Springer-Verlag; 2010.

8. Lee PC, Port JL, Paul S, Stiles BM and Altorki NK. Predictors of long-term survival after resection of esophageal carcinoma with nonregional nodal metastases. Ann Thorac Surg. 2009; 88:186-192; discussion 192-183.

9. Lin CS, Chang SC, Wei YH, Chou TY, Wu YC, Lin HC, Wang LS and Hsu WH. Prognostic variables in thoracic esophageal squamous cell carcinoma. Ann Thorac Surg. 2009; 87:1056-1065. 
10. Tachimori Y, Ozawa S, Numasaki H, Matsubara H, Shinoda $\mathrm{M}$, Toh Y and Udagawa H. Supraclavicular node metastasis from thoracic esophageal carcinoma: A surgical series from a Japanese multi-institutional nationwide registry of esophageal cancer. J Thorac Cardiovasc Surg. 2014; 148:1224-1229.

11. Rizk N, Venkatraman E, Park B, Flores R, Bains MS, Rusch $\mathrm{V}$ and American Joint Committee on Cancer staging s. The prognostic importance of the number of involved lymph nodes in esophageal cancer: implications for revisions of the American Joint Committee on Cancer staging system. J Thorac Cardiovasc Surg. 2006; 132:1374-1381.

12. Tepper J, Krasna MJ, Niedzwiecki D, Hollis D, Reed CE, Goldberg R, Kiel K, Willett C, Sugarbaker D and Mayer R. Phase III trial of trimodality therapy with cisplatin, fluorouracil, radiotherapy, and surgery compared with surgery alone for esophageal cancer: CALGB 9781. J Clin Oncol. 2008; 26:1086-1092.

13. Mariette C, Dahan L, Mornex F, Maillard E, Thomas PA, Meunier B, Boige V, Pezet D, Robb WB, Le Brun-Ly V, Bosset JF, Mabrut JY, Triboulet JP, Bedenne L and Seitz JF. Surgery alone versus chemoradiotherapy followed by surgery for stage I and II esophageal cancer: final analysis of randomized controlled phase III trial FFCD 9901. J Clin Oncol. 2014; 32:2416-2422.

14. Kaklamanos IG, Walker GR, Ferry K, Franceschi D and Livingstone AS. Neoadjuvant treatment for resectable cancer of the esophagus and the gastroesophageal junction: a meta-analysis of randomized clinical trials. Ann Surg Oncol. 2003; 10:754-761.

15. Chirieac LR, Swisher SG, Ajani JA, Komaki RR, Correa AM, Morris JS, Roth JA, Rashid A, Hamilton SR and Wu TT. Posttherapy pathologic stage predicts survival in patients with esophageal carcinoma receiving preoperative chemoradiation. Cancer. 2005; 103:1347-1355.
16. Donahue JM, Nichols FC, Li Z, Schomas DA, Allen MS, Cassivi SD, Jatoi A, Miller RC, Wigle DA, Shen KR and Deschamps C. Complete pathologic response after neoadjuvant chemoradiotherapy for esophageal cancer is associated with enhanced survival. Ann Thorac Surg. 2009; 87:392-398; discussion 398-399.

17. Wang BY, Liu CY, Lin CH, Hsu PK, Hsu WH, Wu YC and Cheng CY. Endoscopic tumor length is an independent prognostic factor in esophageal squamous cell carcinoma. Ann Surg Oncol. 2012; 19:2149-2158.

18. Berger AC, Farma J, Scott WJ, Freedman G, Weiner L, Cheng JD, Wang $\mathrm{H}$ and Goldberg M. Complete response to neoadjuvant chemoradiotherapy in esophageal carcinoma is associated with significantly improved survival. J Clin Oncol. 2005; 23:4330-4337.

19. Wang CC, Cheng JC, Tsai CL, Lee JM, Huang PM, Lin CC, Hsu CH, Hsieh MS, Chang YL and Hsu FM. Pathological stage after neoadjuvant chemoradiation and esophagectomy superiorly predicts survival in patients with esophageal squamous cell carcinoma. Radiother Oncol. 2015; 115:9-15.

20. Park JS, Choi JY, Moon SH, Ahn YC, Lee J, Kim D, Kim $\mathrm{K}$ and Shim YM. Response evaluation after neoadjuvant chemoradiation by positron emission tomography-computed tomography for esophageal squamous cell carcinoma. Cancer Res Treat. 2013; 45:22-30. 